Theories \& Applications, the International Edition

Printed Version : (ISSN 2090-5262)

Online Version : (ISSN 2090-5270)

July 2014, Volume 4, No. 2 Pages (156 - 160)

\title{
The Effect of Cross Training on Role of Microrna-206 as Indicator of Muscle Hypertrophy for Basketball Players.
}

\author{
Fatma Hassan Abd El baset Mourgan \\ Assistant. Professor of Faculty of Physical Education, Helwan University, Egypt.
}

\begin{abstract}
Exercise training induces numerous muscular and cardiovascular changes but the mechanism on the molecular basis is still not understood. microRNA molecules (miRNAs) have been recently identified as essential intracellular mediators may help in muscle cells hypertrophy. miR-206, a muscle-specific miRNA that is up-regulated by exercise affecting muscular hypertrophy. The purpose of this study was to identify The effect of Cross Training to assess miRNA-206 concentrations in basketball players at rest and after a proposed program during transitional period as indicator of muscle hypertrophy for basketball players. Where the (number of weeks training program Proposition 6 weeks, the number of training units 18 and training units time 90 minutes), The researcher used the experimental design with one groups of pre-and post-measurements to a sample of 10 players selected by intentional way. The most important results showed on the effectiveness of the proposed program, Where the cross training have a positive impact on the levels of miRNA-206 after effort either before or after training basketball program with maximum elevation in post-exercise after program To increase the level of physical efficiency. It is concluded that microRNA (miRNA) molecules are essential intracellular mediators of numerous biological processes including angiogenesis, inflammation, and mitochondrial metabolism with resultant muscular hypertrophy. The researcher recommends application the suggested training program because it has a positive effect in improving the skillful performance for juniors basketball.
\end{abstract}

Key words: • Cross Training

- microRNA-206 • muscle hypertrophy

Introduction

Gercise training induces numerous muscular and Cardiovascular changes including mitochondrial synthesis, myocardial remodeling, and angiogenesis. Although such adaptations and their attendant impact on exercise capacity and health outcomes have been well documented, the cellular and molecular mechanisms leading to these changes remain incompletely understood.(13:547554),(1:1121-1128), (12:619-626),(2:3983-3994)

Recently, microRNA molecules (miRNAs) have been identified as essential intracellular mediators of processes inherent in exercise adaptation including angiogenesis (Zhang, 2010), inflammation, mitochondrial metabolism (Davidson-Moncada et al. 2010) (Chan et al. 2009; Dang, 2010), cardiac/skeletal muscle contractile force generation, and tissue hypertrophy (Davidsen et al. 2011).

Most miRNAs are transcribed from DNA as long primary transcripts (pri-miRNA) which are processed by Drosha resulting in pre-miRNA hairpins with a length of about 70 nucleotids. After being transported from nucleus to the cytosol via Exportin-5 the hairpins are cleaved to form miRNA duplexes which again are cleaved to form the mature miRNAs. This short single stranded miRNAs are incorporated into the RNAinduced silencing complex (RISC) which regulates protein expression. (25:22-39)
To date, more than 700 human miRNAs have been identified (and the number is still increasing), possibly regulating $30-60 \%$ of protein encoding genes (Friedman et al., $2009 \&$ Griffiths-Jones et al., 2008). MiRNAs usually are expressed in various cell types but interestingly, some miRNAs are expressed tissue-specific such as miR-1, miR-133a, and miR-206 in muscle (Lee et al., 2008).

(Eccentric) exercise-induced muscle damage involves a series of events starting with mechanical disruption of sarcomeres, followed by impaired excitation-contraction coupling and calcium signaling, and finally, activation of proteolytic pathways related to muscle fiber degradation and repair (Griffiths-Jones et al., 2008). Muscle damage coincides with ultra-structural changes in muscle architecture, loss of muscular strength and muscle soreness. miRNAs play important roles in the plasticity of skeletal muscle in response to hypertrophy but also atrophy signals, whereby local but also systemic inflammatory processes accompany the remodeling of skeletal muscle tissue. However, data especially derived from human studies are sparse, possibly due to the fact that skeletal muscle biopsies are not as easy to access and to investigate as blood.

Up to now it is unproven whether miRNAs are involved in inflammatory processes within skeletal muscle tissue but a small number of striated muscle-specific miRNAs so called MyomiRs have been identified and shown to have an important role in myogenesis, embryonic muscle growth and cardiac function and hypertrophy (Zhang et al., 2010). miR- 
206, a muscle-specific miRNA that is up-regulated by exercise in the intracellular space (Nielsen et al.2010) may be involved in tissue repair and adaptation.

Data defining circulating micro-RNA (c-miRNA) behavior in the settings of acute exercise bouts and sustained exercise training in healthy humans are lacking. Identification of miRNAs specifically regulated by exercise could reveal unique biomarkers of exercise physiology and would lend significant insight into the molecular control of exercise adaptation.

Basketball is a high intensity game of cardio and skill followed by a quick recovery period. Due to this physical requirement conditioning is crucial in the game of basketball. If players are not in top shape, their skill level drops significantly as soon as fatigue sets in. There is nothing more frustrating than losing a game because of fatigue. Basketball players need to be able to run the court at pace consistently during the length of a game. Players need to have a quick first step, read and react to game situations, move in any direction at any time, and jump high a and quickly in rapid succession. To obtain these elements basketball players need a strength base and conditioning base. A player who is well conditioned will achieved the best results through sport specific exercises.(27)

Cross-training, sometimes referred to as circuit training, refers to combining exercises of other disciplines, different than that of the athlete in training. In reference to running, cross-training is when a runner trains by doing another kind of fitness workout such as cycling, swimming, a fitness class or strength training, to supplement their running. It builds strength and flexibility in muscles that running doesn't utilize. It prevents injury by correcting muscular imbalances. And the variety prevents boredom and burnout. Alternative forms of exercise have definite benefits: improved your fitness, injury prevention and rehabilitation, quicker recovery, and boredom busters.(28)

Basketball demands strength, endurance, and flexibility from its participants. Unfortunately, most people overlook the importance of cross training and its impact on a wide range of athletic endeavors. In particular, cross training can be used to enhance one's performance on the court.(29)

The purpose of this study was to assess miRNA-206 concentrations for basketball players at rest and after a proposed basketball game, before and after 6 weeks period of cross training program as indicator of muscle hypertrophy, and the impact in improving the physical working capacity (PWC) and some of physical variables.

\section{Material \& Methods:}

Method of the Research: The researcher used the experimental design with one group of pre-and postmeasurements.

Sample of the Research: The sample included 10 basketball players from El Maadi club, season (2011-2012), its selected by intentionally method. All participants were required to abstain from non-steroidal anti-inflammatory use for at least 7 days prior the experimental study day.

Measurement Tools: Medical Weights for measure the body weight per kilograms- restameter to measure total body length -electronic stopwatch- chalk - measure tape numbered ruler - adhesive tape - Balls medical - Basketball court - cycle argometer.

Measurements of the Research: The research measurements included ( micro RNA levels - The physical working capacity (PWC) - long jump stability - Forward drape -Throw ball medical - vertical jump - Run 400 m Run $4 \mathrm{sec}$ )

The Training Program: The main objective of the training program is develop a standardized training plan using cross training, including a set exercise of (Cardiovascular Endurance - Flexibility - swimming - Agility - Strength Training). and recognize the effect on , and the impact in improving the physical working capacity (PWC) and some of physical variables for basketball players. The training program took 6 weeks and included 18 training units, 3 training units per week, and the time for the training unit was (90 Min), The intensity of the training ranged between $75-90 \%$ of the maximum capacity of the players. The program implemented with the assistance of the team coach under the supervision and implementation of the researcher. Exercise on a cycle argometer to exhaustion was performed on the sampling days.

\section{Plasma sampling:}

Five milliliters of blood was collected before and after working on cycle argometer until fatigue under aseptic conditions in standard anticoagulant (EDTA)-treated vacationer tubes at baseline (prior to exercise testing) and immediately post-exercise testing. All blood samples were centrifuged at $2000 \mathrm{~g}$ for $10 \mathrm{~min}$ to pellet cellular elements immediately after each blood draw. The supernatant plasma was then separated and immediately frozen at -80 ?C till PCR assay.

\section{Mirna Extraction:}

Total RNA extraction was performed using a MicroRNA Extraction Kit (Shanghai Shine Gene Molecular Biotech, Inc., Shanghai, China). Quantitative efficacy and reproducibility of c-miRNA extraction was confirmed by extraction via serial dilutions.

\section{Quantification of microRNA expression:}

To quantify levels of select c-miRNA, standard reverse transcription-quantitative ('real time') polymerase chain reaction (RT-QPCR) was utilized. Specifically, reverse transcription was performed to generate cDNA representing levels of mature c-miRNA molecules.

Fold-change of miRNA 206 species was calculated using the formula specified by the manufacturing company. Specifically, in this formula, $C$ t represents the 'real time' cycle number at which the increase in miRNA probe 
fluorescence is exponential. As reference control for miRNA quantification, $C \mathrm{t}$ values were then subtracted from $\mathrm{Ct}$ values obtained from exogenously added miR-206, as described above. $C t$ values were then compared $(\Delta C \mathrm{t})$ with each athlete's own resting baseline at the pre-season time point (normalized to fold-change of 1).

Statistical analysis:

Results
A Computer program (SPSS version 17) was used to analyze obtained results. Student's Mean, standard deviation of players and t test were used to examine the difference between baseline and after exercise measurements before and after the training program. Probability was assigned significant at $=0.05$.

(Table 1)

Statistical characterization of the in the variables for the growth

\begin{tabular}{|c|c|c|c|}
\hline Variables & Mean \pm SD & Skewness & Kurtosis \\
\hline Age (year) & $\mathbf{1 8 . 8} \pm \mathbf{0 . 6 3}$ & $\mathbf{0 . 1 3 2}$ & $\mathbf{0 . 1 7 9}$ \\
\hline Height $(\mathrm{cm})$ & $\mathbf{1 6 9} \pm 1.41$ & $\mathbf{- 2 . 1 0 0}$ & $\mathbf{- 0 . 7 3 8}$ \\
\hline Weight $(\mathrm{kg})$ & $\mathbf{6 9 . 1} \pm 1.37$ & $\mathbf{0 . 1 0 4}$ & -1.169 \\
\hline
\end{tabular}

Describes tables (1) Skewness may confined between $( \pm 3)$, which indicates that the homogeneity of the sample.

(Table 2)

Difference between (before cross training program compared to after program) in microRNA-206 levels for investigated subjects

\begin{tabular}{|c|c|c|c|c|c|}
\hline \multicolumn{7}{|c|}{ Mean \pm SD } \\
\hline variables & Before & After & $t$ & p & Significance \\
\hline miRNA rest & $607.3 \pm 136.59$ & $622.2 \pm 112.83$ & $\mathbf{0 . 9 9}$ & $\mathbf{0 . 3 4}$ & NS \\
\hline miRNA effort & $677.6 \pm 108.19$ & $\mathbf{8 5 2 . 8} \pm 116.73$ & $\mathbf{6 . 1 6}$ & $\mathbf{0 . 0 0}$ & S \\
\hline Ratio increased & $1.13 \pm 0.09$ & $1.39 \pm 0.15$ & 7.24 & $\mathbf{0 . 0 0}$ & S \\
\hline
\end{tabular}

Although miRNA levels increased numerically after the program with significant increase in ratio of miRNA levels training program in resting, there were no significant indicating adaptation of skeletal muscles to the training differences in results. Effort results are increased after program.

(Table 3)

Significance of differences between (before cross training program compared to after program) in PWC170, and physical variables

\begin{tabular}{|c|c|c|c|c|c|}
\hline \multicolumn{6}{|c|}{ Mean \pm SD } \\
\hline variables & Before & After & $\mathbf{t}$ & $\mathbf{p}$ & Significance \\
\hline $\mathrm{PWC}_{170}$ & $13.74 \pm 3.37$ & $17.88 \pm 4.94$ & 2.89 & 0.022 & $\mathbf{S}$ \\
\hline Vertical Jump & $26.28 \pm 4.76$ & $33.46 \pm 5.83$ & 2.91 & 0.043 & $\mathbf{S}$ \\
\hline Throw ball medical & $2.79 \pm 0.53$ & $3.78 \pm 0.90$ & 3.04 & 0.003 & $\mathbf{S}$ \\
\hline long jump stability & $179.84 \pm 16.75$ & $256.58 \pm 14.84$ & 4.79 & $\mathbf{0 . 0 2 7}$ & $\mathbf{S}$ \\
\hline 400m Run & $126.83 \pm 13.37$ & $97.74 \pm 11.71$ & 3.54 & 0.009 & $\mathbf{S}$ \\
\hline 4 second Run & $16.93 \pm 2.63$ & $21.74 \pm 2.49$ & 3.85 & 0.041 & $\mathbf{S}$ \\
\hline Agility & $19.18 \pm 3.82$ & $24.61 \pm 4.71$ & 2.69 & 0.017 & $\mathbf{S}$ \\
\hline Forward drape & $4.73 \pm 1.01$ & $13.94 \pm 2.70$ & 6.16 & 0.026 & $\mathbf{S}$ \\
\hline
\end{tabular}

Cross-training program lead to increased physical variables with remarkable significant difference in results $(\mathrm{p}<05)$ of $\mathrm{PWC}_{170}$. There was a tendency of improved physical variables . Results before program compared to that obtained after program are represented in table (3).

Discussion 
The present study was proposed to study assays for plasma miRNA-206, the physical working capacity (PWC) and some of physical variables to determine the effects of a cross training program undergone through six successive weeks during transitional period. Also, to investigate cell metabolism after strenuous exercise.

The significant increase in plasma miRNA 206 after exercise and its resistance elevation after about 6 weeks of training suggests that miRNA-206 could possibly be an important tool for monitoring and quantification of cellular adaptation.

This study comprised three main results; the first is the increased 1.13 fold elevation of plasma miRNA-206 after acute exhausting exercise, the second is more increased elevation of miRNA-206 up to 1.39 fold due to the cross training program and third is the developed physical working capacity (PWC) and some of physical variables due to program. These observations suggest a potential role for c-miRNAs as biomarkers of exercise physiology and provide novel insight into the potential regulatory role of $\mathrm{c}$ miRNAin the myriad physiological processes that accompany exercise.

Results from Baggish et al. (2011) study assure these findings as they reported significant changes in circulating miRNAs in response to exercise and demonstrated that these changes may occur in response either to a single acute bout of exhaustive exercise or to sustained exercise training. Thus, these molecules appear integrally linked to both the initial stresses and the longer term adaptations that are characteristic of exercise.

In contrast, up-regulation after chronic exercise training may rely upon modulation of either transcriptional or posttranscriptional processing of intracellular miRNA. Nonspecific release of miRNA from peripheral muscle tissue may also contribute to increases in circulating miRNA (cmiRNA) levels, as strenuous and/or eccentric aerobic exercise can acutely cause microscopic damage to muscle cells. (4:228-233)

Several miRNAs are highly regulated in vivo and in vitro during muscle development and, in turn, regulate muscle differentiation (Baggish et al., 2011). The most-studied miRNAs are miR-133a/b, miR-206, and miR-1, which are induced during differentiation of my oblasts into my tubes (Proske and Allen, 2005) and are collectively referred to as the "memoirs." The memoirs, which are also highly expressed in vivo, may also be regulated during changes in muscle phenotype (Davidsen et al., 2011).

Upon transfection in the continued presence of serum, miR206 markedly up-regulated the percentage of cells expressing myosin heavy chain (MHC) and the musclespecific transcription factor myogenin. In addition, $28 \%$ of MHC positive cells are multinucleated after miR-206 treatment, whereas no multinucleated cells were detected in miR-206 absent control. Therefore, physiological levels of miR-206 induce skeletal myogenesis (Kim et al., 2006).
It appears that myomiRs may be associated with muscle growth and regeneration (Chen et al. 2006; Nakasa $\boldsymbol{e t}$ al. 2009), implicating a potential role in skeletal muscle adaptation to exercise. Indeed, myomiR expression is acutely altered during post-exercise recovery in humans and rats (Drummond et al. 2008; Safdar et al. 2009) while models of muscle overload can significantly alter myomiR expression to new steady state levels (McCarthy \& Esser, 2007).

The cross training is important to build both conditioning and strength bases before working on sports specific drills and exercises. Keeping these two bases strong will make on court training much easier. With these training programs in place can make an individual's basketball performance improve rapidly.(1)

\section{Conclusion \& Recommendations}

The cross training program lead to increased developed physical working capacity (PWC) and some of physical variables and 1.39 fold elevations in miRNA-206. miRNAs are secreted into the bloodstream and that circulating miRNAs (c-miRNAs) may serve important endocrine functions. miRNA expression has previously been associated with fiber type composition. More studies are still required to investigate more microRNAs effect and correlated to the type of exercise.

\section{References}

1. Baggish AL,Wang F,Weiner RB, Elinoff JM, Tournoux F, Boland A, Picard MH, Hutter AM Jr \&Wood MJ (2008): Training-specific changes in cardiac structure and function: a prospective and longitudinal assessment of competitive athletes. $J$ Appl Physiol 104, 1121-1128.

2. Baggish, AL., Hale, A., Weiner, RB., Lewis, GD., Systrom, D., Wang, F., Wang, TJ. and Chan, SY. (2011): Dynamic regulation of circulating microRNA during acute exhaustive exercise and sustained aerobic exercise training, J Physiol 589.16 (2011) pp 398339943983

3. Chan SY, Zhang YY, Hemann C, Mahoney CE, Zweier JL \& Loscalzo J (2009). MicroRNA-210 controls mitochondrial metabolism during hypoxia by repressing the iron-sulfur cluster assembly proteins ISCU1/2, Cell Metab 10, 273-284.

4. Chen JF, Mandel EM, Thomson JM,WuQ, Callis TE, Hammond SM, Conlon FL \&Wang DZ (2006): The role of microRNA-1 and microRNA-133 in skeletal muscle proliferation and differentiation, Nat Genet 38, 228-233.

5. Dang CV ( 2010). Rethinking the Warburg effect with Myc micromanaging glutamine metabolism, Cancer Res 70, 859-862.

6. Davidsen PK, Gallagher IJ, Hartman JW, Tarnopolsky MA, Dela F, Helge JW, Timmons JA \& Phillips SM (2011). High responders to resistance exercise training demonstrate differential regulation of skeletal muscle microRNA expression, J Appl Physiol 110, 309-317. 
7. Davidson-Moncada J, Papavasiliou FN \& TamW(2010): MicroRNAs of the immune system: roles in inflammation and cancer, Ann N Y Acad Sci 1183, 183-194.

8. Drummond, MJ. (2010): MicroRNAs and exerciseinduced skeletal muscle adaptations, J Physiol 588.20 (2010) pp 3849-3850

9. Drummond MJ, McCarthy JJ, Fry CS, Esser KA \& Rasmussen BB (2008): Aging differentially affects human skeletal muscle microRNA expression at rest and after an anabolic stimulus of resistance exercise and essential amino acids, Am J Physiol Endocrinol Metab 295, E1333-E1340.

10. Friedman RC, Farh KK, Burge CB, Bartel DP. Most mammalian mRNAs are conserved targets of microRNAs, Genome Res. 2009, 19: 92-105.

11. Griffiths-Jones S, Saini HK, van Dongen S, Enright AJ. miRBase: tools for microRNA genomics, Nucleic Acids Res. 2008, 36: D154-158.

12. Gute D, Fraga C, Laughlin MH \& Amann JF (1996). Regional changes in capillary supply in skeletal muscle of high-intensity endurance-trained rats, $J \mathrm{Appl}$ Physiol 81, 619-626.

13. Kiessling KH, Pilstrom L, Karlsson J \& Piehl K (1973). Mitochondrial volume in skeletal muscle from young and old physically untrained and trained healthy men and from alcoholics, Clin Sci 44, 547-554.

14. Kim, HK., Lee, YS., Sivaprasad, U., Malhotra, A. and Dutta, A. (2006): Muscle-specifi c microRNA miR-206 promotes muscle differentiation, JCB $17: 5$

15. Lee EJ, Baek M, GusevY, Brackett DJ, Nuovo GJ, Schmittgen TD. Systematic evaluation of microRNA processing patterns in tissues, cell lines, and tumors, Rna. 2008, 14: 35-42.

16. McCarthy JJ. MicroRNA-206: the skeletal musclespecific myomiR. Biochim Biophys Acta. 2008, 1779; 682-691.

17. McCarthy JJ, Esser KA, Peterson CA \& DupontVersteegden EE (2009): Evidence of MyomiR network regulation of $\beta$-myosin heavy chain gene expression during skeletal muscle atrophy, Physiol Genomics 39, 219-226.
18. McCarthy JJ \& Esser KA (2007): MicroRNA-1 and microRNA-133a expression are decreased during skeletal muscle hypertrophy, J Appl Physiol 102, 306313.

19. Mccarthy JJ. (2008): MicroRNA-206: the skeletal muscle-specific myomiR. Biochim Biophys, Acta 2008;1779:682-691.

20. McCarthy JJ, Esser KA, Peterson CA \& DupontVersteegden EE (2009). Evidence of MyomiR network regulation of $\beta$-myosin heavy chain gene expression during skeletal muscle atrophy, Physiol Genomics 39, 219-226.

21. Nakasa T, Ishikawa M, ShiM, Shibuya H, Adachi N \& Ochi M (2009), J Cell MolMed

22. Nielsen, S., Scheele, C., Yfanti, C., Akerstr"om, T., Nielsen, AR., Pedersen BK. and Laye, M. (2010): Muscle specific microRNAs are regulated by endurance exercise in human skeletal muscle, $J$ Physiol 588.20 (2010) pp 4029-4037 4029

23. Proske U, Allen TJ. Damage to skeletal muscle from eccentric exercise. Exerc Sport Sci Rev. 2005, 33: 98104

24. Safdar A, Abadi A, Akhtar M, Hettinga BP \& Tarnopolsky MA (2009): miRNA in the regulation of skeletal muscle adaptation to acute endurance exercise in C57B1/6J male mice, PLoS ONE 4, e5610.

25. Wessner B, Gryadunov-Masutti L, Tschan H, Bachl $\mathbf{N} \&$ Roth $\mathbf{E}$ (2010). Is there a role for microRNAs in exercise immunology? A synopsis of current literature and future developments, Exerc Immunol Rev 16, 22 39.

26. Zhang Y, Liu D, Chen X, Li J, Li L, Bian Z, Sun F, Lu J, Yin Y, Cai X, Sun Q,Wang K, Ba Y,Wang Q,Wang D, Yang J, Liu P, Xu T, Yan Q, Zhang J, Zen K \& Zhang CY (2010). Secreted monocytic miR150 enhances targeted endothelial cell migration, $\mathrm{Mol}$ Cell 39, 133-144.

27. http://crosstrainingtips.com/cross-training/build-strongbases-for-basketball/\#

28. http://www.runnersworld.com/tag/cross-training

29. http://www.personaltrainertoday.com/cross-trainingand-basketball 